\title{
AXILLOCORONARY BYPASS FOR SEVERELY ATHEROSCLEROTIC AORTA IN CORONARY ARTERY BYPASS GRAFTING
}

\author{
Johannes Bonatti, MD, Herbert Hangler, MD, Herwig Antretter, MD, and Ludwig C. Müller, MD, Innsbruck, Austria
}

Stroke after coronary artery bypass grafting (CABG) is one of the most feared complications in cardiac operations. Cannulation and clamping of a severely atherosclerotic ascending aorta can lead to cerebral embolization of atheromatous debris and should therefore be absolutely avoided. Alternatives to ascending aortic cannulation and crossclamping have included proximal descending aortic or femoral artery cannulation with placement of arterial in situ grafts ${ }^{1}$ or innominate artery-to-coronary artery vein grafts $^{2}$ on the fibrillating or beating heart. Replacement of the ascending aorta with a synthetic graft in deep hypothermic cardiocirculatory arrest and implantation of vein grafts into the prosthesis has been applied clinically as well. $^{3}$

In this brief communication we report a case of severely atherosclerotic ascending aorta detected during CABG. As an alternative to aortic crossclamping, an axillocoronary saphenous vein graft was placed to the right coronary artery (RCA) in addition to a left internal thoracic artery in situ graft to the left anterior descending artery (LAD).

From the University Clinic of Surgery, Cardiac Surgery, Anichstrasse 35, A-6020 Innsbruck, Austria.

Received for publication Sept. 16, 1997; accepted for publication Oct. 29, 1997.

Address for reprints: Johannes Bonatti, MD, University Clinic of Surgery, Cardiac Surgery, Anichstrasse 35, A-6020 Innsbruck, Austria.

J Thorac Cardiovasc Surg 1998;115:956-7

Copyright (c) 1998 by Mosby, Inc.

$0022-5223 / 98 \$ 5.00+0 \quad \mathbf{1 2 / 5 4 / 8 7 2 2 3}$
The procedure was carried out on the beating heart with cardiopulmonary bypass and proximal aortic arch cannulation. The postoperative course was uneventful, no neurologic deficit was detectable, and the axillocoronary bypass showed excellent flow.

Clinical summary. A 73-year-old woman was scheduled to undergo CABG for two-vessel disease. Cardiovascular risk factors included arterial hypertension and hypercholesterolemia. An anterior wall myocardial infarction was reported 6 years before CABG and LAD percutaneous transluminal coronary angioplasty shortly thereafter. The patient complained of angina on exertion; coronary angiography showed an $80 \%$ proximal LAD stenosis and a $99 \%$ proximal RCA stenosis.

After median sternotomy and harvesting of the internal thoracic artery, the pericardium was opened. Inspection of the ascending aorta showed a heavily atherosclerotic vessel with numerous calcified plaques on the anterior wall. By gentle palpation and epiaortic echography a soft spot on the proximal aortic arch could be detected for arterial cannulation.

The right axillary artery was exposed between the pectoralis major and deltoid muscle above the anterior axillary fold (Fig. 1). After systemic heparinization, the axillary artery was clamped and incised. A piece of saphenous vein harvested from the right leg was sutured to the axillary artery using 6-0 Prolene polypropylene (Ethicon, Inc., Somerville, N.J.). The right pleura was opened, and a wide hole in the intercostal space adjacent to the axillary artery anastomotic site was created digitally. The saphenous vein graft was brought through this intercostal opening. Adequate blood flow was detected. Car- 


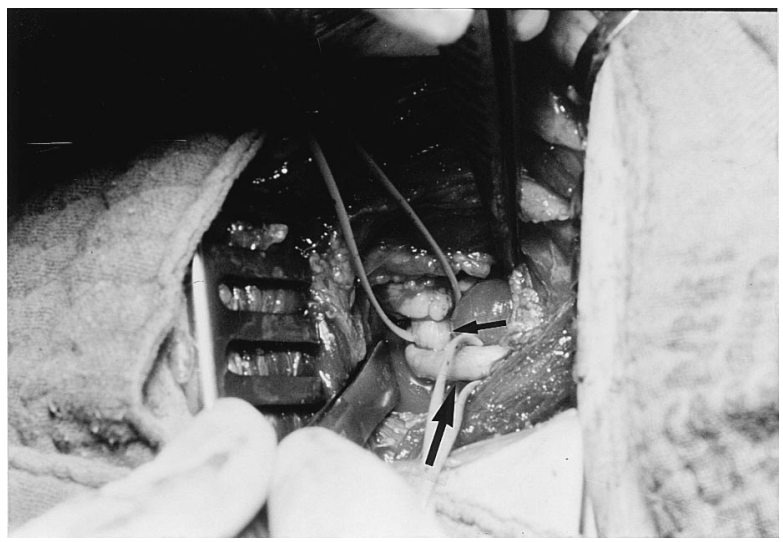

Fig. 1. The axillocoronary bypass (large arrow) seen through the axillary incision ( small arrow $=$ medial cord of the brachial plexus).

diopulmonary bypass was done after venous cannulation of the right atrium and arterial cannulation of the proximal aortic arch. The patient was cooled to $32^{\circ} \mathrm{C}$ and a left ventricular vent was inserted through the right superior pulmonary vein. Blood flow in the RCA was interrupted by two 3-0 Prolene snaring sutures. The target vessel was opened longitudinally, the axillocoronary vein graft was trimmed to adequate length, and an end-to-side anastomosis was performed on the beating heart using 7-0 Prolene sutures (Fig. 2). Vein graft length required was 34 $\mathrm{cm}$. The left internal thoracic artery was then sutured to the LAD as an in situ graft. The procedure was completed uneventfully.

No significant problems occurred during the postoperative course. The patient was extubated 12 hours after the operation and discharged from the intensive care unit on the first postoperative day. No signs of myocardial ischemia were present, and bleeding was negligible. Neurologic evaluation did not reveal any central nervous system deficit and no brachial plexus lesion. Arterial perfusion of the right upper extremity always remained normal.

For evaluation of the axillocoronary bypass flow continuous wave Doppler evaluation was chosen. The graft could easily be assessed from the origin from the axillary artery to the entrance into the adjacent intercostal space. A typical diastolic flow was detected, and a graft flow of 56 $\mathrm{ml} / \mathrm{min}$ was calculated. A restudy by coronary angiography carried out 6 weeks after the operation showed a patent axillocoronary vein graft with a kink- and tension-free course.

Discussion. The procedure described in comparison to other alternative operations for an untouchable aorta seems to offer several advantages. First, saphenous vein coronary artery bypass is technically easier to perform than all arterial revascularization. ${ }^{1}$ Second, performance

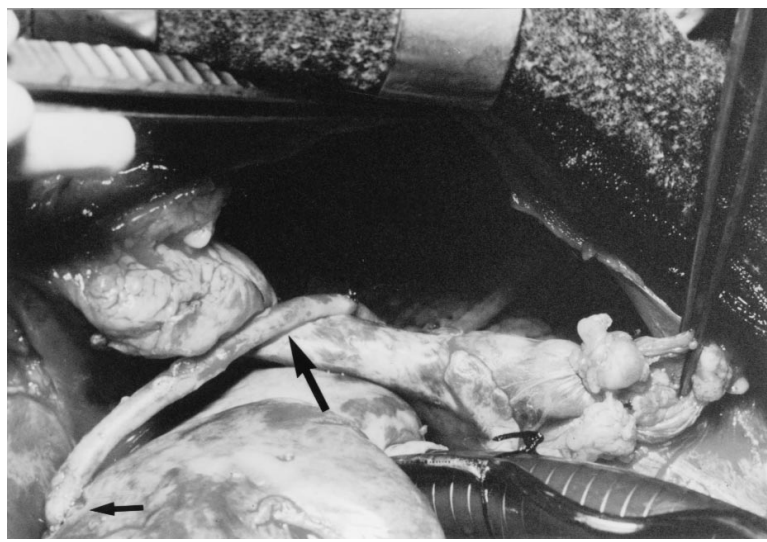

Fig. 2. The axillocoronary bypass graft (large arrow) sutured end to side to the right coronary artery (small arrow $=$ anastomosis).

of an innominate artery-to-coronary artery bypass puts the patient at a certain risk for stroke by placement of a side wall clamp that might lead to embolization of atherosclerotic debris. ${ }^{2}$ This is not the case in axillocoronary bypass grafting. The risk of arm ischemia seems to be present in both innominate artery-to-coronary bypass grafting and axillocoronary bypass grafting, but cerebral ischemia should be reduced by the latter technique. Low perioperative stroke rates have been demonstrated after replacement of the ascending aorta by a vascular prosthesis and connection of venous bypass grafts to the synthetic graft. $^{3}$ This procedure, however, requires induction of deep hypothermic cardiocirculatory arrest, which by itself carries the risk of postoperative neurologic deficits. Axillocoronary bypass grafting as described in this communication only involves moderate hypothermia and does not directly jeopardize the central nervous system.

In summary, a technically simple extraanatomic variation of coronary venous bypass grafting is demonstrated that can potentially reduce the risk of stroke in cases of untouchable aorta during CABG.

\section{REFERENCES}

1. Suma H. Coronary artery bypass grafting in patients with calcified ascending aorta: aortic no-touch technique. Ann Thorac Surg 1989;48:728-30.

2. Weinstein G, Killen DA. Innominate artery-coronary artery bypass graft in patient with calcific aortitis. J Thorac Cardiovasc Surg 1980;79:312-3.

3. Wareing TH, Davila Roman VG, Daily BB, Murphy SF, Schechtman KB, Barzilai B, et al. Strategy for the reduction of stroke incidence in cardiac surgical patients. Ann Thorac Surg 1993;55:1400-7. 